\title{
Synthesis and antioxidant activity evaluations of melatonin based analogue indole-hydrazide/ hydrazone derivatives
}

\author{
AD Yılmaz ${ }^{*}$, H Shirinzadeh ${ }^{1}$, T Coban², S Suzen ${ }^{1}$, S Ozden ${ }^{1}$ \\ From International Conference for Healthcare and Medical Students 2011 \\ Dublin, Ireland. 4-5 November 2011
}

\section{Introduction}

Harmful effects of free radicals to the human body have been studied over the last decade. Overproduction of the free radicals can be responsible for tissue injuries that cause many health problems which include cancer, aging, heart diseases, neurological disorders, Alzheimer's disease, Huntington disease and so on. Melatonin (MLT), the main secretory product of the pineal gland is a wellknown antioxidant and free radical scavenger. It is a neurohormone produced from the amino acid tryptophan. In our earlier studies, new MLT-based analogues with changes in the 5-methoxy and 2-acylaminoethyl groups of MLT were synthesized and tested for their in vitro antioxidant potency in the DPPH, superoxide dismutase and lipid peroxidation (LP) assays.

\section{Methods}

In this study 5-chloroindole hydrazide/hydrazone derivatives were synthesized from 5-chloroindole-3-carboxaldehyde and phenyl hydrazine derivatives. All the compounds characterized and in vitro antioxidant activity was investigated against MLT and BHT.

\section{Results}

The synthesized compounds were tested for their antioxidant activities using DPPH and superoxide radical scavenging and LP inhibitory activity tests. Ten of the synthesized compounds showed strong inhibitory effect on the superoxide radical scavenging assay. Almost all the tested compounds possessed strong scavenging activity against the DPPH radical scavenging activity with IC50 values (2 to $60 \mu \mathrm{M})$.

${ }^{1}$ Department of Pharmaceutical Chemistry, Faculty of Pharmacy, Ankara

University, Turkey

Full list of author information is available at the end of the article

\section{Conclusions}

MLT has redox properties because of the presence of an electron-rich aromatic ring system, which allows the indoleamine to easily function as an electron donor12,13,31. It is possible that making the indole ring more stable electronically helped to act as a better electro donor. MLT scavenges the radicals via nitrogen centred radical, the indolyl (or melatonyl) cation radical32. Introduction of an imine group in to the side chain increased the stability of the indole molecule by helping the delocalization of the electrons. This might help to have high free radical scavenging activity in the synthesized.

\section{Author details}

'Department of Pharmaceutical Chemistry, Faculty of Pharmacy, Ankara University, Turkey. ${ }^{2}$ Department of Pharmaceutical Toxicology, Faculty of Pharmacy, Ankara University, Turkey.

Published: 9 July 2012

doi:10.1186/1753-6561-6-S4-P28

Cite this article as: Yllmaz et al:: Synthesis and antioxidant activity evaluations of melatonin based analogue indole-hydrazide/hydrazone derivatives. BMC Proceedings 2012 6(Suppl 4):P28.

( 2012 Y Ilmaz et al; licensee BioMed Central Ltd. This is an Open Access article distributed under the terms of the Creative Commons 